\title{
BRCA1 and BRCA2 mutations sensitize to chemotherapy in patient-derived pancreatic cancer xenografts
}

\author{
I Lohse ${ }^{1}$, A Borgida ${ }^{2}$, P Cao ${ }^{1}$, M Cheung ${ }^{1}$, M Pintilie ${ }^{1}$, T Bianco ${ }^{2,3}$, S Holter $^{2}$, E lbrahimov ${ }^{1,2}$, R Kumareswaran ${ }^{1}$, \\ R G Bristow ${ }^{1}$, M-S Tsao ${ }^{1,4,5}$, S Gallinger ${ }^{2,3}$ and D W Hedley ${ }^{*, 1,3,6}$ \\ ${ }^{1}$ Ontario Cancer Institute and Campbell Family Cancer Research Institute, Princess Margaret Cancer Centre, University Health \\ Network, Toronto, Ontario, Canada M5G 2M9; ${ }^{2}$ Mount Sinai Hospital, Joseph and Wolf Lebovic Health Complex, Toronto, \\ Ontario, Canada M5G 2M9; ${ }^{3}$ Translational Research Initiative in Pancreas Cancer, Ontario Institute for Cancer Research, Toronto, \\ Ontario, Canada M5G 2M9; ${ }^{4}$ Department of Pathology, University Health Network, Toronto, Ontario, Canada M5G 2M9; \\ ${ }^{5}$ Department of Laboratory Medicine and Pathobiology, University of Toronto, Toronto, Ontario, Canada M5G 2M9 and \\ ${ }^{6}$ Department of Medical Oncology and Haematology, Princess Margaret Cancer Centre, Toronto, Ontario, Canada M5G 2M9
}

Background: Germline mutations of the BRCA tumour suppressors have been associated with increased risk of pancreatic cancer. Clinical evidence suggests that these patients may be more sensitive to treatment with cisplatin. As the frequency of germline BRCA mutations is low, definitive experimental data to support the clinical observations are still missing.

Methods: We tested gemcitabine and cisplatin sensitivity of four BRCA1 and BRCA2 mutant and three BRCA1 and BRCA2 wild-type (WT) patient-derived pancreatic cancer xenografts.

Results: We observed treatment sensitivity to gemcitabine and cisplatin in the BRCA WT and mutant models. The BRCA1 and BRCA2 mutant xenografts were significantly more sensitive to cisplatin although these models also showed sensitivity to gemcitabine. The BRCA1 and BRCA2 WT models showed sensitivity to gemcitabine but not cisplatin. Treatment sensitivity in the xenograft models closely resembled treatment response in the corresponding patients.

Discussion: We have characterised a panel of xenografts derived from pancreatic cancer patients carrying germline BRCA mutations, and shown that their genetic features resemble the patient donor. Our results support further clinical testing of treatment regimens combining gemcitabine and platinum drugs in this patient population, as well as preclinical research aiming to identify mechanisms of cisplatin resistance in BRCA mutant pancreatic cancers.

It has been estimated that up to $10 \%$ of pancreatic cancers have a hereditary component with autosomal dominant transmission of mutations in tumour suppressors as the prevalent genetic basis for increased risk (Ghadirian et al, 1991; Fernandez et al, 1994; Klein et al, 2001; Bartsch et al, 2004; Grant et al, 2014). Although germline mutations in several known cancer susceptibility genes have been implicated in increased risk of pancreatic cancer (Hruban et al, 2010), mutations in the breast cancer early onset (BRCA1 \& BRCA2) tumour suppressor genes are currently the best characterised and appear to be to be responsible for $\sim 15 \%$ of familial cases (Couch et al, 2007). Patients with BRCA2 mutation have been reported to have an overall 3.5 -fold risk of developing pancreatic cancer compared with the general population (The Breast Cancer Linkage Consortium, 1999).

The BRCA tumour suppressors are involved in the repair of DNA double-strand breaks (DSB) and DNA cross-linking damage induced by DNA-damaging agents through the homologous recombination (HR) pathway. The BRCA1 and BRCA2 are 
localised to the nucleus in response to DNA damage leading to the formation of RAD51 foci and subsequent DNA damage repair (Venkitaraman, 2001; Gudmundsdottir and Ashworth, 2006). The lack of RAD51 foci implies defects in the HR pathways (Gudmundsdottir and Ashworth, 2006). In addition to its function in HR, BRCA2 function is essential for non-HR repair (Venkitaraman, 2001; Gudmundsdottir and Ashworth, 2006).

Clinical data in ovarian cancer show that patients with BRCA1 and BRCA2 mutations show higher response rates to treatment with cisplatin and other DNA-damaging agents resulting in improved outcome (Dann et al, 2012; Muggia and Safra, 2014). Similar observations have been made in case reports of patients with pancreatic cancer, suggesting that BRCA1 and BRCA2 mutations may sensitise to treatment with platinum drugs (Lowery et al, 2011; Sonnenblick et al, 2011). This is supported by preclinical studies using established pancreatic cancer cell lines (van der Heijden et al, 2005), and also by a large retrospective analysis of 71 pancreatic cancer patients with germline BRCA1 and BRCA2 mutations, where it was reported that patients with stage 3/ 4 disease who were treated with a platinum-containing regimen had a median survival of 22 months, compared with 9 months for those who did not receive platinum (Golan et al, 2014).

There are important unanswered questions concerning the optimum design of platinum-containing treatment protocols for this group of patients, as well as the role of newer agents such as inhibitors of poly-ADP-ribose polymerase (PARP) that have shown early promise in other cancer patients carrying germline BRCA mutations. Owing to the relatively low frequency of these mutations in pancreatic cancer patients, it is difficult to address these questions efficiently using prospective clinical trials. As primary xenografts derived from pancreatic cancers appear to maintain characteristics of the patient donor (Chang et al, 2011; Lohse et al, 2014), they offer an alternative, 'near-clinical' approach for the development and testing of treatment protocols for patients with BRCA mutations. However, the extent to which primary xenografts derived from BRCA mutant pancreatic cancers maintain the genetic and phenotypic features of the patient donor remains unclear, and is of particular concern given the potential for genomic instability conferred by BRCA mutations. These questions are addressed in the present paper.

\section{MATERIALS AND METHODS}

Patients. Subcutaneous and orthotopic tumours of seven primary xenografts, designated as Ontario Cancer Institute Pancreas (OCIP) 19, 23, 28, 167, 217, 232 and A1, were established from pancreatectomy samples superfluous to patient diagnosis or ascites samples using a protocol approved by the University Health Network Research Ethics Board. Informed consent was obtained from all the participating patients.

Patients OCIP28 (http://www.ncbi.nlm.nih.gov/clinvar/ RCV000044800/), OCIP217 (http://www.ncbi.nlm.nih.gov/ clinvar/RCV000019244/), OCIP232 and OCIPA1 had clinically relevant, deleterious germline mutations in BRCA1 and BRCA2 (Table 1). The presence of the patient mutation was confirmed in the xenografts by Sanger sequencing.

Primary patient-derived xenografts. Animal experiments were carried out using protocols approved by the University Health Network (UHN) Animal Care Committee under the guidelines of the Canadian Council on Animal Care. Primary xenografts were established from pancreatectomy samples as previously described (Chang et al, 2011; Lohse et al, 2014). Briefly, tumour fragments were implanted subcutaneously into the flank of 4-5-week-old non-obese diabetic severe combined immune-deficient mice (NOD/SCID). All the models used in this study showed first- generation growth and $100 \%$ take rate from the third passage on and can be regrown from cryopreserved tumour fragments. The xenograft models closely resemble the morphology of the patient specimen (Chang et al, 2011; Lohse et al, 2014) and show stable growth rates over multiple passages. Subcutaneous tumours were measured using callipers, and volume calculated according to the formula width ${ }^{2} \times$ length $\times 0.5$.

Treatments were started when tumour volume reached $\sim 150 \mathrm{~mm}^{3}$. Animals were treated with $4 \mathrm{mg} \mathrm{kg}^{-1}$ cisplatin (Sigma-Aldrich, Oakville, ON, Canada) weekly intraperitoneal or $100 \mathrm{mg} \mathrm{kg}^{-1}$ gemcitabine (Accord Healthcare Inc, North Harrow, UK) biweekly intraperitoneal for 30 days. Animals were killed when tumours reached humane end point according to institutional guidelines.

For acute treatments, animals were treated with a single dose of $4 \mathrm{mg} \mathrm{kg}^{-1}$ cisplatin and tumours were excised for analysis $24 \mathrm{~h}$ after treatment.

Histological analysis. For BRCA1, BRCA2 and RAD51 staining, tumours were excised, fixed and paraffin embedded. Paraffin tissue sections were cut, dried and dewaxed. Following peroxidase block and antigen retrieval, sections were incubated for $1 \mathrm{~h}$ at room temperature with antibodies against RAD51 (Santa Cruz, Dallas, TX, USA, sc-8349, 1:100), BRCA1 (Santa Cruz, sc-1021, 1:100) and BRCA2 (Santa Cruz, sc-1818, 1:50). This was followed with AlexaFluor 555 labelled secondary (Invitrogen, Burlington, ON, Canada) for $60 \mathrm{~min}$. DAPI (Sigma-Aldrich, Oakville, ON, Canada) was applied for $5 \mathrm{~min}$. The slides were then air dried and stored at $4{ }^{\circ} \mathrm{C}$. Images were acquired using the Olympus IX81 Spinning Disk Confocal Microscope (Richmond Hill, ON, Canada). Tumours containing nuclei with more than five nuclei per cells were counted as positive (Fraser et al, 2011).

To determine the level of tumour hypoxia, mice were injected intraperitoneally with the 2-nitroimidazole hypoxia marker EF5, $30 \mathrm{mg} \mathrm{kg}^{-1}, 3 \mathrm{~h}$ before being killed (Lord et al, 1993; Koch, 2002). Tumours were excised and processed as described previously (Chang et al, 2011; Lohse et al, 2014). Sections were labelled with primary antibodies to $\alpha$-smooth muscle Actin ( $\alpha$-SMA), (DAKO, Glostrup, Denmark, clone 1A4, 1:400), CD31 (Santa Cruz, sc-1506, 1:1000) overnight. Biotinylated anti-mouse IgG incubations were carried out followed by streptavidin biotin detection system (Signet Pathology System, Deham, MA, USA). For EF5 staining, slides were incubated in biotinylated EF5 antibody (obtained from Dr Cameron Koch, University of Pennsylvania, Philadelphia, PA, USA, 1:250) (Lord et al, 1993; Koch, 2002). Sections were scanned at $\times 20$ resolution using an Aperio Scanscope XT scanner (Aperio Technologies, Vista, CA, USA). Images were analysed using the Aperio ImageScope software ver. 11.1.2.752, positive pixel count algorithm (PPC). Necrotic areas were excluded from the analysis.

For double-fluorescent staining, paraffin tissue sections were cut, dried and dewaxed. After antigen retrieval and serum block, sections were incubated at room temperature with $\gamma \mathrm{H} 2 \mathrm{AX}$ (EMD Millipore, Etobicoke, ON, Canada, clone JBW301, 1:1000) and cleaved caspase-3 (CC3) (Cell Signaling, Danvers, MA, USA, \#9661, 1/200) cocktail overnight. Secondary antibodies (goat anti-mouse AlexaFluor 647 and goat anti-rabbit AlexaFluor 555, Invitrogen, 1/100) were applied for $1 \mathrm{~h}$ at room temperature. Sections were stained with DAPI for $10 \mathrm{~min}$ at room temperature and air dried.

Images of the entire section were obtained using a multilaser scanner (TS4000; Huron Technologies, Waterloo, ON, Canada) at $0.5 \mu \mathrm{m}$ per pixel. Region of tumour, necrosis, stroma, folds were specified, creating a training rule-set for tissue recognition. Cellular analysis included nucleus identification and separation; objects $<10 \mu \mathrm{m}^{2}$ were excluded. Individual nuclear mean intensities for $\gamma \mathrm{H} 2 \mathrm{AX}$ and $\mathrm{CC} 3$ channels were recorded. A threshold was 
Table 1. Primary pancreatic cancer resection specimen

\begin{tabular}{|c|c|c|c|c|c|c|c|c|c|}
\hline Model & Sex & Diagnosis & Grade & $\begin{array}{l}\text { Clinical } \\
\text { stage }^{a}\end{array}$ & $\begin{array}{l}\text { Pathology } \\
\text { stage }^{\text {a }}\end{array}$ & Surgery & Recurrence & $\begin{array}{c}\text { Germline BRCA } \\
\text { mutation }^{\mathrm{b}}\end{array}$ & $\begin{array}{l}\text { Survival } \\
\text { (days) }\end{array}$ \\
\hline OCIP19 & M & $\begin{array}{l}\text { Ductal } \\
\text { ADC }\end{array}$ & G2 & T3NxMO & T3N1b & $\mathrm{Y}$ & Local & WT & 562 \\
\hline OCIP23 & M & $\begin{array}{l}\text { Ductal } \\
\text { ADC }\end{array}$ & G3 & T3NxMO & T3N1b & Y & Distant & WT & 249 \\
\hline OCIP28 & $\mathrm{F}$ & $\begin{array}{l}\text { Ductal } \\
\text { ADC }\end{array}$ & G1 & T4NOMO & T3NO & $Y$ & & $\begin{array}{l}\text { BRCA2 c.6174delT } \\
\text { p.S1982Rfs }\end{array}$ & $2047^{c}$ \\
\hline OCIP167 & M & $\begin{array}{l}\text { Ductal } \\
\text { ADC }\end{array}$ & G2 & T2NxM0 & T3N1b & $\mathrm{Y}$ & Distant & WT & 1150 \\
\hline OCIP217 & $\mathrm{F}$ & $\begin{array}{l}\text { Ductal } \\
\text { ADC }\end{array}$ & G3 & T4NOM1 & T2NO & $Y$ & Distant & $\begin{array}{l}\text { BRCA1 } \\
\text { c.4327C }>\text { T, } \\
\text { p.R1443X }\end{array}$ & 777 \\
\hline OCIP232 & M & $\begin{array}{l}\text { Ductal } \\
\text { ADC }\end{array}$ & G2 & TxNxM1 & T3N1b & Y & Distant & $\begin{array}{l}\text { BRCA2 } \\
\text { c.3393delC } \\
\text { p.L1059X }\end{array}$ & 681 \\
\hline OCIPA1 & M & $\begin{array}{l}\text { Ductal } \\
\text { ADC }\end{array}$ & G3 & T3N1M1 & I & $N^{d}$ & $\mathrm{~N}$ & $\begin{array}{l}\text { BRCA2 } \\
\text { c. } 1736 \mathrm{~T}>\mathrm{G}, \\
\text { p.L579X }\end{array}$ & 355 \\
\hline \multicolumn{10}{|c|}{ 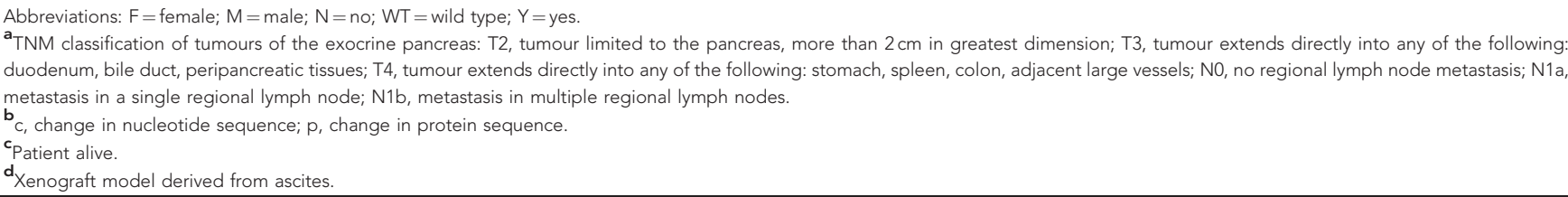 } \\
\hline
\end{tabular}

determined by mean $+5 \times$ s.d. intensity in the $\gamma \mathrm{H} 2 \mathrm{AX}$ and CC3 channels. No image processing was carried out before the analysis.

For p53 (Vector Labs, Burlington, ON, Canada, 1:250), p16 (Abcam, Cambridge, MA, USA, 1:100) and Smad4 (Abcam, 1:100) stainings, paraffin tissue sections were cut, dried and dewaxed. Following peroxidase block and antigen retrieval sections were incubated for $1 \mathrm{~h}$ at room temperature with primary antibodies. After incubation in biotinylated anti-rabbit IgG (Vector Labs, 1:200) followed by HRP labelling reagent (Signet Pathology System Inc) for $30 \mathrm{~min}$, immunoreactivities were revealed by incubation in Nova Red substrate (Vector Labs) for $5 \mathrm{~min}$ and counterstained in Mayer's haematoxylin. Slides were scanned at $\times 20$ resolution using an Aperio Scanscope XT scanner, and the percentage of positive pixels determined using the Aperio Imagescope software (Vs.11.1.2.752, Aperio Technologies).

Statistical analysis. The tumour growth data were analysed using mixed effect modelling which accounts for the correlations among the measurements of the same model. The tumour volume was transformed to the linear scale (power 1/3) to stabilise the variance of the residuals. The survival percentages for the in vivo data were calculated using the Kaplan-Meier technique and the curves were tested for significance using the log-rank test. The changes in DNA damage as measured by $\gamma \mathrm{H} 2 \mathrm{AX}$ and $\mathrm{CC} 3$ were analysed by applying the mixed effect modelling to mitigate the existent correlations between the measurements of the same model. Both markers needed to be log-transformed to obtain residuals distributed normally.

\section{RESULTS}

BRCA1 and BRCA2 patient-derived xenografts. For this study, we used a set of four pancreatic cancer xenografts established from patients with known germline mutations in the BRCA 1 and BRCA2 genes (Table 1). The BRCA1 and BRCA2 mutants express truncated proteins with C-terminal deletions (Figure 1A and B). These models were compared with three wild-type (WT) xenografts without germline or somatic mutations in BRCA1 or
BRCA2. The control models were selected to match the BRCA mutants in terms of growth rate as indicated by the time elapsed between two passages, magnitude of hypoxia as shown by staining for the hypoxia marker EF5 and stromal content as indicated by $\alpha$ SMA staining (Supplementary Figure 1A-C).

The OCIP217 model expresses a truncated version of BRCA1 that has lost the BRCT domain involved in BRCA1 recruitment to the DNA damage site and part of the ATM-binding domain (Figure 1A). Loss of heterozygosity was demonstrated in the patient-derived xenograft when compared with the patient specimen.

The OCIP28, OCIP232 and OCIPA1 models express truncated versions of BRCA2 of different lengths (Figure 1B). The OCIP28 model expresses a 2002 amino acid (AA) protein that has lost its nuclear localisation sequence (NLS), the oligonucleotide binding domains (OB) as well as part of the RAD51-binding domain. Although the patient tumour that OCIP28 was derived from retained the WT BRCA2 allele, loss of heterozygosity occurred in the patient-derived xenograft. OCIP232 carries a germline mutation that gives rise to a 1059AA version of BRCA2 that has lost most of the RAD51-binding domain. In addition, the OCIP232 xenograft model carries a somatic mutation (c.G8909A, p.W2970X). This mutation results in the expression of a truncated BRCA2 protein, while maintaining the RAD51-binding domain has lost the NLS and OB domains. OCIPA1 expresses the shortest BRCA2 protein with 579AA. The WT allele was retained in the OCIPA1 xenograft.

To evaluate BRCA1 and BRCA2 function, animals were treated with a single dose of cisplatin and BRCA1, BRCA2 and RAD51 expression evaluated $24 \mathrm{~h}$ after treatment.

The WT models displayed an increase in both BRCA1 and BRCA2 expression in response to treatment with cisplatin (Supplementary Figure 1D and E) and cytoplasmic and nuclear foci. Similar staining patterns were observed in the mutant xenografts stained for BRCA1 with the exception of OCIPA1, which did not show any BRCA1 staining. The OCIP28 tumours stained for BRCA2 showed no increase in staining in response to cisplatin treatment, while staining levels were similar to WT controls. The OCIP217, 232 and A1 models displayed a mostly 
A Human BRCA1
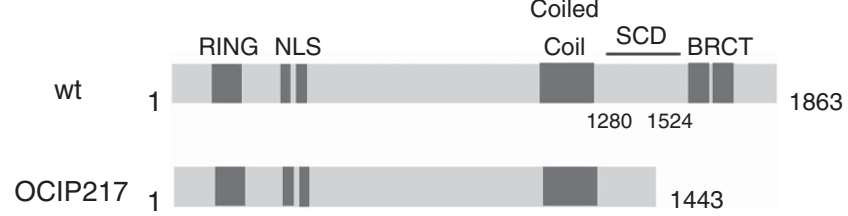

B Human BRCA2

$\mathrm{OB}$
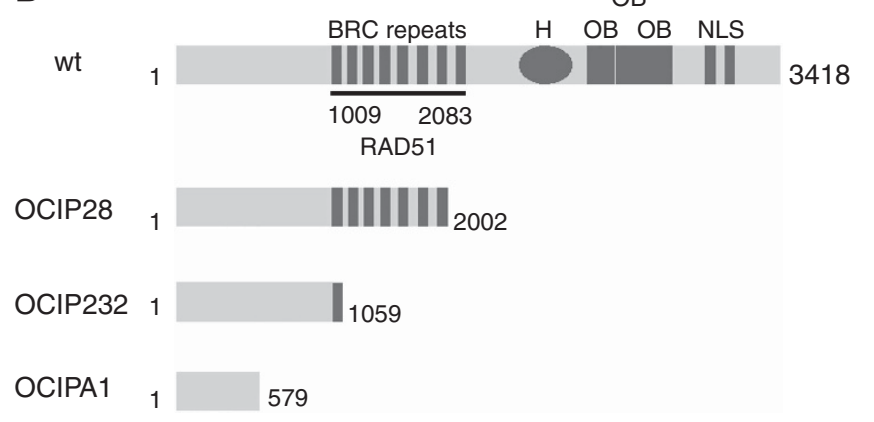

C

OCIP

19

23
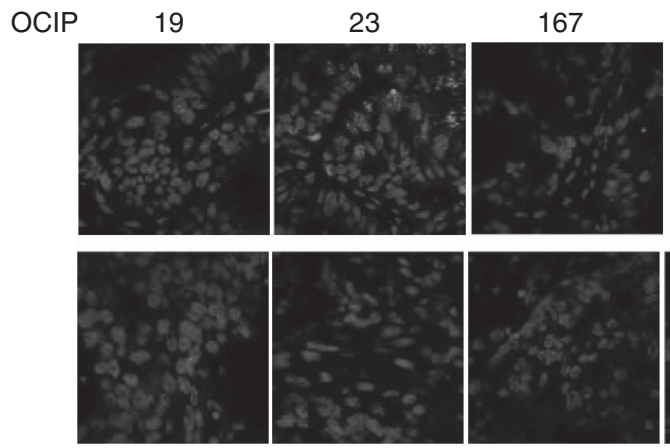

OCIP

28

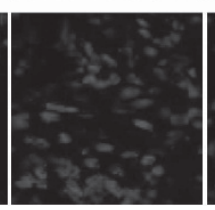

217

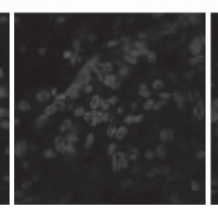

232

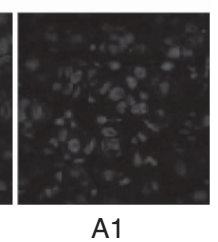

Figure 1. BRCA1 and BRCA2 mutants. (A) The BRCA1 amino terminus contains a RING domain and the nuclear localisation sequence (NLS). The carboxyl terminus contains the coiled coil domain that facilitates BRCA2 binding, a SQ/TQ cluster domain (SCD) that contains 10 possible ATM phosphorylation sites and a BRCT domain, which is associated with BRCA1 recruitment to the DNA damage site. The mutation found in OCIP217 leads to the expression of a truncated BRCA1 protein that has lost the BRCT domain and parts of the ATM $\mathrm{SCD}$. (B) BRCA2 contains eight BRC repeats between amino acids (AA) 1009 and 2083 that binds RAD51. The carboxyl terminus contains helical domain $(\mathrm{H})$, three oligonucleotide binding (OB) domains, which facilitates binding to both single-stranded and double-stranded DNA, and an NLS. All BRCA2 mutants express truncated proteins that have lost the carboxyl terminus. While OCIP28 maintains seven BRC repeats, OCIP232 and OCIPA1 have lost the RAD51-binding domains.

Cellular localisation of (C) RAD51(red) in sections of xenograft tumours treated with vehicle or cisplatin. DAPI (blue) was used to visualise the cell nucleus.

diffuse cytoplasmic staining pattern (Supplementary Figure 1D and E).

Consistent with a functional DNA double-strand break (DSB) repair system, all the WT models displayed RAD51 foci in response to treatment with cisplatin (Figure 1C). No RAD51 foci were observed in the BRCA1 and BRCA2 mutant xenografts in response to treatment with cisplatin (Figure 1C).

The WT and BRCA mutant xenografts were characterised for mutations in Kras, p53, p16 and Smad4 (Supplementary Table 1). All the models show mutation in Kras codon 12. Three of the seven models (OCIP23, 232, A1) show mutations in p53. Loss of p16 was observed in OCIP28, 167, 232 and A1. With the exception of OCIP167 and OCIPA1, Smad4 was expressed in all the models.

BRCA1 and BRCA2 mutations sensitise to treatment. To test sensitivity to gemcitabine (Gem) and cisplatin (Cis) in vivo, tumour-bearing mice were treated with 4 weeks of gemcitabine, cisplatin or vehicle.

The BRCA WT xenograft models OCIP19, OCIP23 and OCIP167 show significantly reduced tumour growth $(P<0.0001)$ in response to treatment with gemcitabine compared with vehicle or cisplatin-treated tumours (Figure 2A). Although treatment with cisplatin significantly reduced tumour growth in OCIP167 $(P<0.0001)$, the effect is less pronounced. OCIP19 $(P=0.016)$ and OCIP23 $(P<0.0001)$ showed little response to cisplatin treatment. Treatment with gemcitabine consistently resulted in a significant increase in survival in OCIP19 $(P=0.029)$ and OCIP167 $(P=0.0011)$ while treatment with cisplatin only led to a mild increase in survival $(P=0.013$ and 0.0011 , respectively for OCIP19 and OCIP167). Neither treatment increased survival in OCIP23 (Figure 2B).

In contrast, both gemcitabine and cisplatin significantly reduced tumour growth in all the BRCA mutant xenograft models $(P<0.0001)$. Both treatments are significantly more potent in BRCA mutant xenografts than BRCA WT models $(P<0.0001)$. In the BRCA2 mutant OCIP28 and the BRCA1 mutant OCIP217, treatment with either drug resulted in complete regression of the tumours (Figure 2A), although regrowth occurred in some of the treated mice. Both gemcitabine and cisplatin significantly prolonged survival compared with untreated control $(P<0.0001)$, with some treated mice dying of old age without any palpable tumours (Figure 2B). The BRCA2 mutant OCIP232 and OCIPA1 models also showed prolonged growth inhibition and survival in response to either treatment $(P<0.0001)$. Neither treatment led to significant changes in body weight (Supplementary Figure 2).

BRCA1/2 mutation result in the accumulation of DNA damage. Tumours treated with a single dose of cisplatin or vehicle were stained for $\gamma \mathrm{H} 2 \mathrm{AX}$ and CC3 to examine DNA damage and apoptosis in response to treatment. Tumours were excised $24 \mathrm{~h}$ after treatment. The BRCA mutant xenografts (OCIP28, OCIP217, OCIP232 and OCIPA1) showed a significant accumulation of DNA damage $(P<0.0001)$ as measured by $\gamma \mathrm{H} 2 \mathrm{AX}$ staining that was not observed in the control xenografts (OCIP19, OCIP23 and OCIP167; Figure 3A). Even though the BRCA2 mutant xenograft models showed a significant accumulation of DNA damage, no significant increase in CC3 staining was observed $(P=0.57$; Figure $3 \mathrm{~B}$ ). This was also observed in OCIP217. Alternatively, the control tumours (OCIP19, OCIP23 and OCIP167) displayed a mild but nonsignificant increase in CC3 staining $(P=0.58)$ in response to treatment with cisplatin.

BRCA1 and BRCA2 patients. All the four BRCA mutant xenografts were derived from patients whose mutation was known before implantation, and their clinical features, response to treatment and survival are summarised in Table 1 and Table 2. All of these patients responded to platinum-based chemotherapy. The donor of OCIP28 presented with an unresectable tumour owing to involvement of the superior mesenteric artery. There was a major response to cisplatin combined with gemcitabine, and the patient was then able to undergo curative-intent surgery, (followed by postoperative treatment with radiotherapy and capecitabine and remains alive more than 5 years after surgery. The patient from which OCIP217 was derived showed a complete and sustained response of a solitary liver metastasis following cisplatin plus gemcitabine treatment (no regrowth once chemotherapy was discontinued for 6 months) and a major response of the primary tumour (Figure 2C). She subsequently underwent distal pancreatectomy and wedge resection at the site of the liver lesion (no viable 

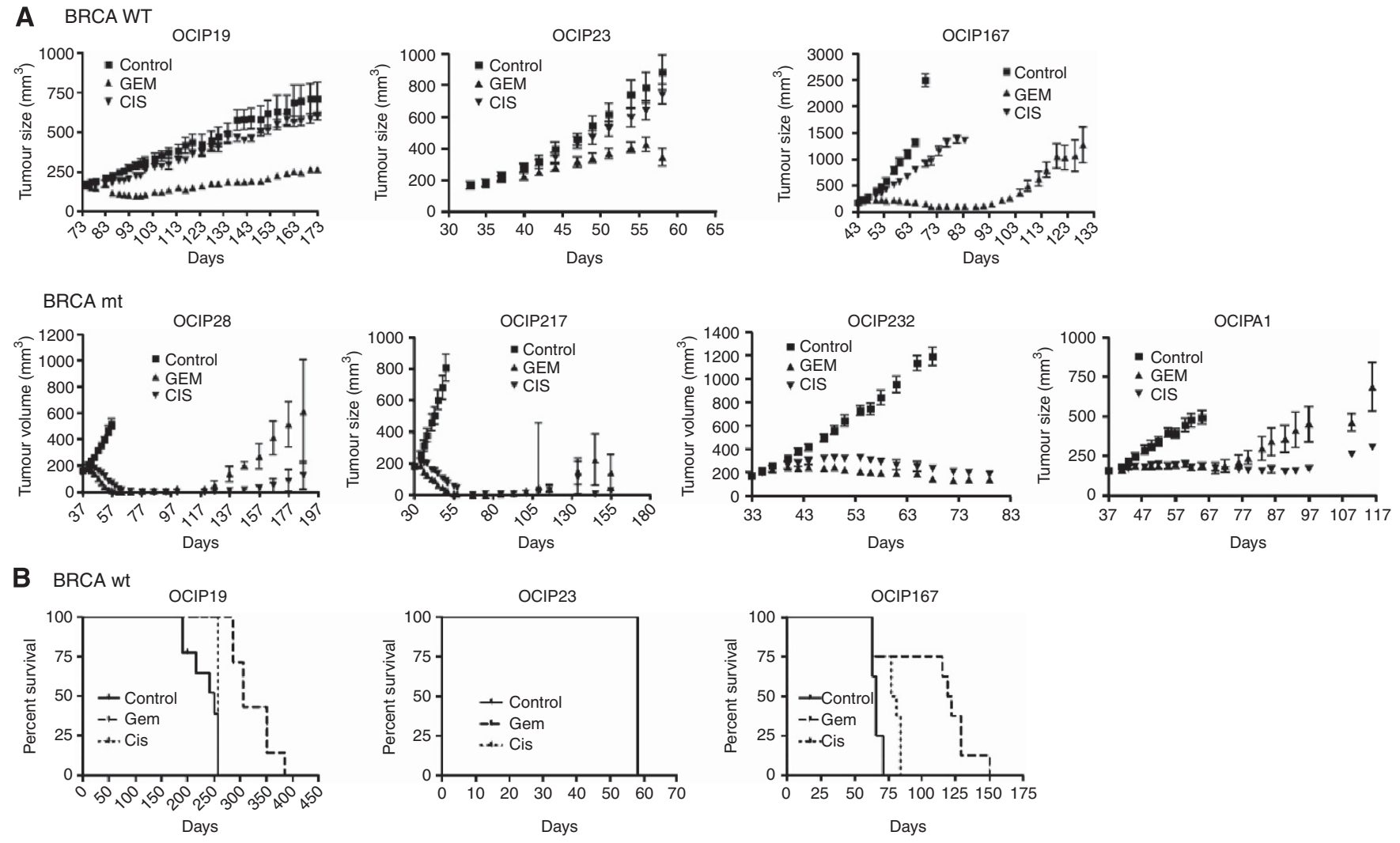

seram
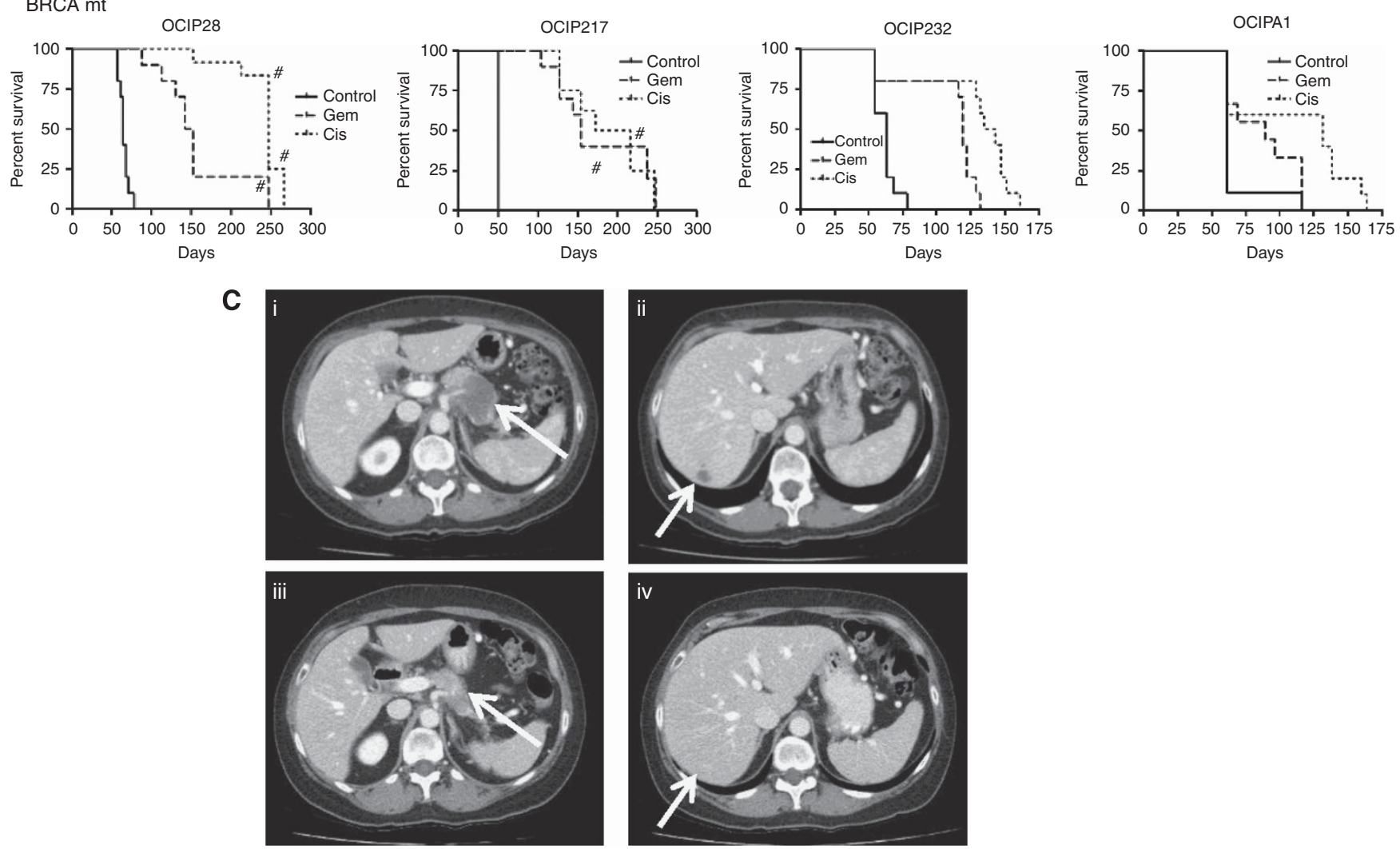

Figure 2. Mutations in BRCA1 and BRCA2 increase treatment sensitivity and survival. (A) Mice were treated for 4 weeks with cisplatin, gemcitabine or vehicle, and tumour volume was evaluated three times a week; mt, mutant. (B) Survival in response to treatment was measured until mice either reached the humane tumour end point or their natural life span in the case of no recurrence of the tumour after treatment. Error bars represent s.d. \# indicates no tumours detected. Mice were killed due to old age. (C) CT scans for patient from which OCIP217 was derived. Primary tumour in the body of pancreas with involvement of coeliac axis and splenic artery (i, arrow) and solitary liver metastasis (ii, arrow). Major response to gemcitabine and cisplatin with marked shrinkage of primary tumour (iii, arrow), and radiologic complete response of liver metastasis (iv, arrow). 

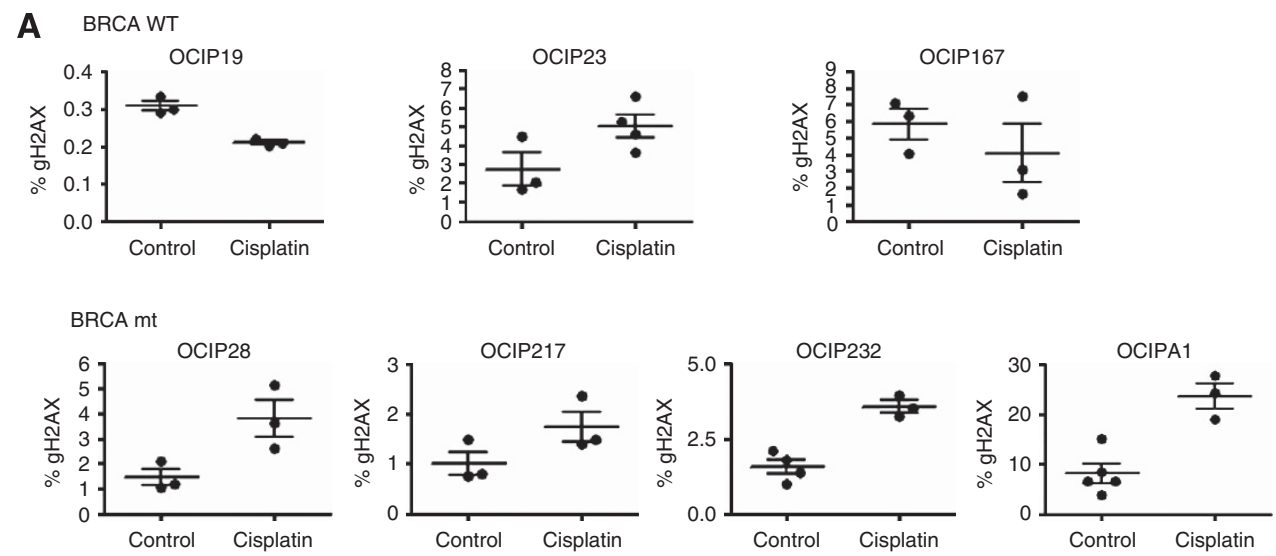

B BRCA WT
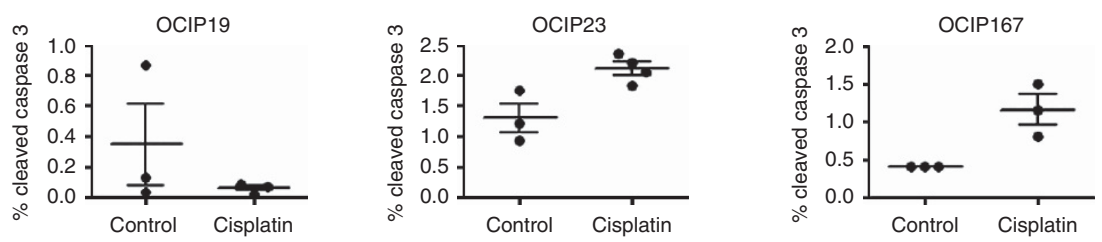

BRCA mt
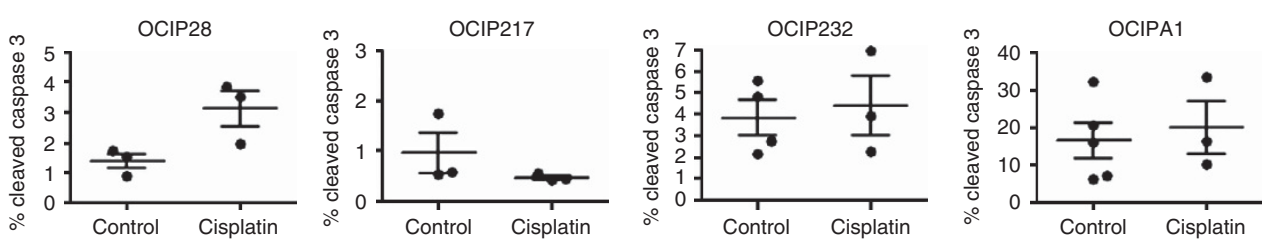

Figure 3. BRCA1 and BRCA2 mutations result in $\gamma \mathrm{H} 2 \mathrm{AX}$ foci accumulation in response to cisplatin treatment. Section of mice treated with a single dose of cisplatin or vehicle were stained for $(\mathbf{A}) \gamma \mathrm{H} 2 \mathrm{AX}$ and (B) CC3. BRCA mutant (mt) models display a significant increase in $\gamma \mathrm{H} 2 \mathrm{AX}$ foci that was not observed in the WT models $24 \mathrm{~h}$ after treatment. Cleaved caspase-3 staining, however, was not increased in the BRCA mt models.

Error bars represent s.d.

\begin{tabular}{|c|c|c|c|}
\hline \multicolumn{4}{|c|}{ Treatment } \\
\hline Model & RT & Chemo & Response \\
\hline \multirow[t]{2}{*}{ OCIP19 } & $\mathrm{Y}$ & $\mathrm{Gem}^{\mathrm{a}}$ & No evidence of disease \\
\hline & & Gem-RT ${ }^{\mathrm{a}}$ & No evidence of disease \\
\hline OCIP23 & $\mathrm{N}$ & $\mathrm{Gem}^{\mathrm{a}}$ & Disease progression \\
\hline \multirow[t]{2}{*}{ OCIP28 } & Y & Gem-Cis ${ }^{b}$ & Partial response \\
\hline & & Capecitabine $^{\mathbf{a}}$ & No evidence of disease \\
\hline \multirow[t]{2}{*}{ OCIP167 } & $\mathrm{N}$ & $\mathrm{Gem}^{\mathrm{a}}$ & No evidence of disease \\
\hline & & $\begin{array}{l}\text { 5FU-irinotecan- } \\
\text { oxaliplatin }^{c}\end{array}$ & Stable disease \\
\hline \multirow[t]{3}{*}{ OCIP217 } & Y & Gem-Cis ${ }^{b}$ & Partial response \\
\hline & & $\mathrm{Gem}-\mathrm{Cis}^{\mathrm{c}}$ & Stable disease \\
\hline & & Carboplatin-paclitaxel & Disease progression \\
\hline \multirow[t]{3}{*}{ OCIP232 } & $\mathrm{N}$ & $\mathrm{Gem}^{\mathrm{a}}$ & No evidence of disease \\
\hline & & $\begin{array}{l}\text { 5FU-irinotecan- } \\
\text { oxaliplatin-leucovorin }^{c}\end{array}$ & $\begin{array}{l}\text { Initial response, progressed after } \\
6 \text { months }\end{array}$ \\
\hline & & Veliparib ${ }^{c}$ & Disease progression \\
\hline \multirow[t]{2}{*}{ OCIPA1 } & Y & $\mathrm{Gem}^{\mathrm{b}}$ & Disease progression \\
\hline & & Gem-Cis ${ }^{c}$ & $\begin{array}{l}\text { Mixed response (primary: response; } \\
\text { liver met: progression) }\end{array}$ \\
\hline \multicolumn{4}{|c|}{$\begin{array}{l}\text { Abbreviations: Cis = cistplatin; Gem = gemcitabine; } N=\text { no; } R T=\text { radiotherapy; } Y=\text { yes. } \\
\text { a Adjuvant. } \\
\text { b Neoadjuvant. } \\
{ }_{\text {Palliative. }}\end{array}$} \\
\hline
\end{tabular}

tumour was found in the liver wedge), but developed peritoneal metastases 3 months later. These were initially controlled using cisplatin plus gemcitabine, but the disease then became platinum refractory and the patient died. The donor of OCIP232 underwent Whipple resection followed by adjuvant gemcitabine, but developed liver metastases less than 1 year post surgery. He was then treated using the FOLFIRINOX protocol (5-fluorouracil, irinotecan and oxaliplatin), showed an initial response, but the disease became refractory after 8 months. The patient was briefly enrolled in a clinical trial of the PARP inhibitor velaparib, but came off study after 2 weeks because of declining performance and died. The donor of OCIPA1 presented with locally advanced disease, showed an initial response to gemcitabine chemoradiation, but then developed malignant ascites (from which the xenograft was established) and osteoblastic metastases. Treatment was changed to cisplatin plus gemcitabine upon identification of a germline BRCA2 mutation. The patient experienced a dramatic but shortlived clinical response, with resolution of ascites and pain, but then developed leptomeningeal metastases and died 4 months later. Because of the difference in grade, clinical stage and treatments given in this small patient series, we did not attempt a statistical analysis for the correlation of xenograft and patient data or treatment efficacy.

\section{DISCUSSION}

Germline mutations in BRCA1 and BRCA2 result in defects in BRCA-mediated HR, which is important for the maintenance of 
genome stability (Venkitaraman, 2001; Gudmundsdottir and Ashworth, 2006). These mutations predispose to breast and ovarian cancers (Foulkes and Shuen, 2013; Kobayashi et al, 2013), and are associated with increased risk of pancreatic cancer (Fernandes et al, 1994; Klein et al, 2001; Bartsch et al, 2004; Grant et al, 2014). In addition to a role in tumour development, BRCA1 and BRCA2 mutations are associated with sensitivity to platinum drugs and other DNA-damaging agents in ovarian cancers (Dann et al, 2012; Muggia and Safra, 2014), and there are anecdotal reports that this is also the case in pancreatic cancers (Lowery et al, 2011; Sonnenblick et al, 2011). Additional support for this comes from a recent retrospective study of 71 pancreatic cancer patients with germline BRCA1 and BRCA2 mutations that reported superior overall survival for the patients who were treated with platinum, compared with those who were not treated with platinum (Golan et al, 2014). However, the clinical development of treatment protocols for these patients is hampered by their relative rarity. To address this, we studied cisplatin activity in a series of primary xenografts obtained from platinum-naive pancreatic cancer patients, compared with a matched group of BRCA WT control tumours, and we made a number of novel observations.

With the exception of the BRCA2 mutant xenograft OCIP28, in which there was loss of heterozygosity, all the four xenografts derived from patients with germline BRCA mutations maintained the genetic features seen in the donor. They displayed a range of phenotypic features that have been linked to aggressive growth, including doubling time, stroma density and hypoxia that were similar to the control group of primary xenografts that lacked BRCA mutations. Importantly, RAD51 repair foci were observed in the control tumours $24 \mathrm{~h}$ after cisplatin treatment, but not in the four BRCA mutant xenografts, confirming their functional deficiency in DSB repair.

As expected, all the four BRCA mutant models were sensitive to cisplatin, which produced long-term control of established OCIP28 and OCIP217 tumours. Interestingly, these tumours also showed comparable sensitivity to gemcitabine. In contrast, cisplatin was essentially inactive against the BRCA WT xenografts, although gemcitabine produced some degree of growth inhibition in all the three models, with pronounced tumour shrinkage and prolonged survival in OCIP167.

This is consistent with observations recently published by Waddell et al (Waddell et al, 2015) who analysed whole genomes of 100 pancreatic ductal adenocarcinomas. Similar to the results presented in this study, PDX models carrying a germline BRCA2 mutation showed high sensitivity to treatment with both cisplatin and gemcitabine. Although Waddell et al used both agents at their maximum-tolerated dose, we used significantly lower doses in our treatments $\left(140 \mathrm{mg} \mathrm{kg}^{-1}\right.$ vs $100 \mathrm{mg} \mathrm{kg}^{-1}$ gemcitabine, $6 \mathrm{mg} \mathrm{kg}^{-1}$ vs $4 \mathrm{mg} \mathrm{kg}^{-1}$ cisplatin). Similar growth delay in response to treatment was observed in both studies despite the difference in dose, suggesting that the dose can be adapted to reduce side effects without loss of treatment efficacy.

It has been proposed that the typically dense stroma of pancreatic cancer affords a barrier to drug penetration (Olive et al, 2009), although this was not supported by recent studies (Rhim et al, 2014; Sherman et al, 2014). There was no obvious correlation between stroma density and treatment sensitivity in these models.

The differential sensitivity of the BRCA WT xenografts to gemcitabine and cisplatin is consistent with gemcitabine as the more clinically active drug. In contrast, all the four BRCA mutant xenografts were highly sensitive to both drugs, with long-term complete regression of established tumours seen in some cases following treatment with either agent. This finding confirms the clinical impression that pancreatic cancers in patients with germline BRCA mutations may be unusually sensitive to platinum-containing compounds. Less attention has been paid to their sensitivity to gemcitabine, which also causes DSBs (Plunkett et al, 1995; Jones et al, 2014). Within the constraints of the small sample size, there was no correlation between tumour growth rate, stroma density and the level of hypoxia and response to either drug.

All the four patients with germline BRCA mutations had the mutation identified before the establishment of the xenografts and treatment with platinum. The response of the xenografts roughly tracks that seen in the corresponding patient, with the donors of the two most-responsive xenografts, OCIP28 and 217, both achieving downstaging with gemcitabine plus cisplatin chemotherapy to undergo curative-intent surgery. It is noteworthy that the donor of OCIP28 remains disease-free after more than 5 years, whereas that of OCIP217 showed complete response of a solitary liver metastasis and over $50 \%$ shrinkage of a $6-\mathrm{cm}$ tumour in the body of the pancreas. In contrast, the donor of the least-responsive xenograft, OCIPA1, rapidly developed cisplatin resistance and survived for only 4 months. With the exception of the OCIP28 donor, resistance to platinum-containing treatment developed in all the patients. It can be speculated that this occurred, in part, due to the emergence of a clone of HR-proficient cancer cells, or to unrelated mechanisms of acquired drug resistance involving, for example, drug accumulation or detoxification pathways.

In summary, we have characterised a panel of primary xenografts derived from pancreatic cancer patients carrying germline BRCA mutations, and have shown that their genetic features and platinum sensitivity closely recapitulate those seen in the patient donor. These models also show greater sensitivity to gemcitabine compared with a matched series of BRCA WT tumours, which we believe is important and under-appreciated when considering future trials of drug combinations. These findings support ongoing preclinical research using these models to identify mechanisms for the emergence of cisplatin resistance in BRCA mutant pancreatic cancers, as well as the evaluation of treatment protocols that incorporate additional agents such as PARP inhibitors.

\section{ACKNOWLEDGEMENTS}

This work was supported by the Farb Family Fund, Princess Margaret Cancer Foundation, the Ontario Ministry of Health and Long-Term Care (OMHLTC) and grants from the Ontario Institute for Cancer Research and the Canadian Cancer Society Research Institute (CCSRI). The views expressed do not necessarily reflect those of the OMHLTC.

CONFLICT OF INTEREST

The authors declare no conflict of interest.

\section{REFERENCES}

Bartsch DK, Kress R, Sina-Frey M, Grützmann R, Gerdes B, Pilarsky C, Heise JW, Schulte KM, Colombo-Benkmann M, Schleicher C, Witzigmann H, Pridöhl O, Ghadimi MB, Horstmann O, von Bernstorff W, Jochimsen L, Schmidt J, Eisold S, Estévéz-Schwarz L, Hahn SA, Schulmann K, Böck W, Gress TM, Zügel N, Breitschaft K, Prenzel K, Messmann H, Endlicher E, Schneider M, Ziegler A, Schmiegel W, Schäfer H, Rothmund M, Rieder H (2004) Prevalence of familial pancreatic cancer in Germany. Int J Cancer 110(6): 902-906.

Chang Q, Juristica I, Do T, Hedley DW (2011) Hypoxia predicts aggressive growth and spontaneous metastasis formation from orthotopicallygrown primary xenografts of human pancreatic cancer. Cancer Res 71(8): 3110-3120. 
Couch FJ, Johnson MR, Rabe KG, Brune K, de Andrade M, Goggins M, Rothenmund H, Gallinger S, Klein A, Petersen GM, Hruban RH (2007) The prevalence of BRCA2 mutations in familial pancreatic cancer. Cancer Epidemiol Biomarkers Prev 16(2): 342-346.

Dann RB, DeLoia JA, Timms KM, Zorn KK, Potter J, Flake 2nd DD, Lanchbury JS, Krivak TC (2012) BRCA1/2 mutations and expression: response to platinum chemotherapy in patients with advanced stage epithelial ovarian cancer. Gynecol Oncol 125(3): 677-682.

Fernandez E, La Vecchia C, D’Avanzo B, Negri E, Franceschi S (1994) Family history and the risk of liver, gallbladder, and pancreatic cancer. Cancer Epidemiol Biomarkers Prev 3(3): 209-212.

Foulkes WD, Shuen AY (2013) In brief: BRCA1 and BRCA2. J Pathol 230(4): 347-349.

Fraser M, Harding SM, Zhao H, Coackley C, Durocher D, Bristow RG (2011) MRE11 promotes AKT phosphorylation in direct response to DNA double-strand breaks. Cell Cycle 10(13): 2218-2232.

Ghadirian P, Boyle P, Simard A, Baillargeon J, Maisonneuve P, Perret C (1991) Reported family aggregation of pancreatic cancer within a population-based case-control study in the Francophone community in Montreal, Canada. Int J Pancreatol 10(3-4): 183-196.

Golan T, Kanji ZS, Epelbaum R, Devaud N, Dagan E, Holter S, Aderka D, Paluch-Shimon S, Kaufman B, Gershoni-Baruch R, Hedley D, Moore MJ, Friedman E, Gallinger S (2014) Overall survival and clinical characteristics of pancreatic cancer in BRCA mutation carriers. Br J Cancer 111(6): $1132-1138$.

Grant RC, Selander I, Connor AA, Selvarajah S, Borgida A, Briollais L, Petersen GM, Lerner-Ellis J, Holter S, Gallinger S (2015) Prevalence of germline mutations in cancer predisposition genes in patients with pancreatic cancer. Gastroenterology 148: 556-564.

Gudmundsdottir K, Ashworth A (2006) The roles of BRCA1 and BRCA2 and associated proteins in the maintenance of genomic stability. Oncogene 25(43): 5864-5874.

Hruban RH, Canto MI, Goggins M, Schulick R, Klein AP (2010) Update on familial pancreatic cancer. Adv Surg 44: 293-311.

Jones RM, Kotsantis P, Stewart GS, Groth P, Petermann E (2014) BRCA2 and RAD51 promote double-strand break formation and cell death in response to gemcitabine. Mol Cancer Ther 13(10): 2412-2421.

Klein AP, Hruban RH, Brune KA, Petersen GM, Goggins M (2001) Familial pancreatic cancer. Cancer J 7(4): 266-273.

Kobayashi H, Ohno S, Sasaki Y, Matsuura M (2013) Hereditary breast and ovarian cancer susceptibility genes. Oncol Rep 30(3): 1019-1029.

Koch CJ (2002) Measurement of absolute oxygen levels in cells and tissues using oxygen sensors and 2-nitroimidazole EF5. Methods Enzymol 352: $3-31$.

Lohse I, Lourenco C, Ibrahimov E, Pintilie M, Tsao MS, Hedley DW (2014) Assessment of hypoxia in the stroma of patient-derived pancreatic tumor xenografts. Cancers 6: 459-471.

Lord EM, Harwell L, Koch CJ (1993) Detection of hypoxic cells by monoclonal antibody recognizing 2-nitroimidazole adducts. Cancer Res 53: $5721-5726$.

Lowery M, Shah MA, Smyth E, Epstein A, Segal A, Rosengarten O, Isacson R, Drukker L, Keinan A, Rachkiman M, Reissman P, Gabizon A, Kelsen D, O’Reilly EM (2011) A 67-year-old woman with BRCA 1 mutation associated with pancreatic adenocarcinoma. J Gastrointest Cancer 42(3): $160-164$.

Muggia F, Safra T (2014) 'BRCAness' and its implications for platinum action in gynecologic cancer. Anticancer Res 34(2): 551-556.

Olive KP, Jacobetz MA, Davidson CJ, Gopinathan A, McIntyre D, Honess D, Madhu B, Goldgraben MA, Caldwell ME, Allard D, Frese KK, Denicola G,
Feig C, Combs C, Winter SP, Ireland-Zecchini H, Reichelt S, Howat WJ, Chang A, Dhara M, Wang L, Rückert F, Grützmann R, Pilarsky C, Izeradjene K, Hingorani SR, Huang P, Davies SE, Plunkett W, Egorin M, Hruban RH, Whitebread N, McGovern K, Adams J, Iacobuzio-Donahue C, Griffiths J, Tuveson DA (2009) Inhibition of Hedgehog signaling enhances delivery of chemotherapy in a mouse model of pancreatic cancer. Science 324(5933): 1457-1461.

Plunkett W, Huang P, Xu YZ, Heinemann V, Grunewald R, Gandhi V (1995) Gemcitabine: metabolism, mechanisms of action, and self-potentiation. Semin Oncol 22(4 Suppl 11): 3-10.

Rhim AD, Oberstein PE, Thomas DH, Mirek ET, Palermo CF, Sastra SA, Dekleva EN, Saunders T, Becerra CP, Tattersall IW, Westphalen CB, Kitajewski J, Fernandez-Barrena MG, Fernandez-Zapico ME, Iacobuzio-Donahue C, Olive KP, Stanger BZ (2014) Stromal elements act to restrain, rather than support, pancreatic ductal adenocarcinoma. Cancer Cell 25(6): 735-747.

Sherman MH, Yu RT, Engle DD, Ding N, Atkins AR, Tiriac H, Collisson EA, Connor F, Van Dyke T, Kozlov S, Martin P, Tseng TW, Dawson DW, Donahue TR, Masamune A, Shimosegawa T, Apte MV, Wilson JS, Ng B, Lau SL, Gunton JE, Wahl GM, Hunter T, Drebin JA, O’Dwyer PJ, Liddle C, Tuveson DA, Downes M, Evans RM (2014) Vitamin D receptormediated stromal reprogramming suppresses pancreatitis and enhances pancreatic cancer therapy. Cell 159(1): 80-93.

Sonnenblick A, Kadouri L, Appelbaum L, Peretz T, Sagi M, Goldberg Y, Hubert A (2011) Complete remission, in BRCA2 mutation carrier with metastatic pancreatic adenocarcinoma, treated with cisplatin based therapy. Cancer Biol Ther 12(3): 165-168.

The Breast Cancer Linkage Consortium (1999) Cancer risks in BRCA2 mutation carriers. J Nat Cancer Inst 91: 1310-1316.

van der Heijden MS, Brody JR, Dezentje DA, Gallmeier E, Cunningham SC, Swartz MJ, DeMarzo AM, Offerhaus GJ, Isacoff WH, Hruban RH, Kern SE (2005) In vivo therapeutic responses contingent on Fanconi anemia/ BRCA2 status of the tumor. Clin Cancer Res 11(20): 7508-7515.

Venkitaraman AR (2001) Functions of BRCA1 and BRCA2 in the biological response to DNA damage. J Cell Sci 114(Pt 20): 3591-3598.

Waddell N, Pajic M, Patch AM, Chang DK, Kassahn KS, Bailey P, Johns AL, Miller D, Nones K, Quek K, Quinn MC, Robertson AJ, Fadlullah MZ, Bruxner TJ, Christ AN, Harliwong I, Idrisoglu S, Manning S, Nourse C, Nourbakhsh E, Wani S, Wilson PJ, Markham E, Cloonan N, Anderson MJ, Fink JL, Holmes O, Kazakoff SH, Leonard C, Newell F, Poudel B, Song S, Taylor D, Waddell N, Wood S, Xu Q, Wu J, Pinese M, Cowley MJ, Lee HC, Jones MD, Nagrial AM, Humphris J, Chantrill LA, Chin V, Steinmann AM, Mawson A, Humphrey ES, Colvin EK, Chou A, Scarlett CJ, Pinho AV, Giry-Laterriere M, Rooman I, Samra JS, Kench JG, Pettitt JA, Merrett ND, Toon C, Epari K, Nguyen NQ, Barbour A, Zeps N, Jamieson NB, Graham JS, Niclou SP, Bjerkvig R, Grützmann R, Aust D, Hruban RH, Maitra A, Iacobuzio-Donahue CA, Wolfgang CL, Morgan RA, Lawlor RT, Corbo V, Bassi C, Falconi M, Zamboni G, Tortora G, Tempero MA. Australian Pancreatic Cancer Genome InitiativeGill AJ, Eshleman JR, Pilarsky C, Scarpa A, Musgrove EA, Pearson JV, Biankin AV, Grimmond SM (2015) Whole genomes redefine the mutational landscape of pancreatic cancer. Nature 518(7540): 495-501.

This work is published under the standard license to publish agreement. After 12 months the work will become freely available and the license terms will switch to a Creative Commons AttributionNonCommercial-Share Alike 4.0 Unported License.

Supplementary Information accompanies this paper on British Journal of Cancer website (http://www.nature.com/bjc) 